\title{
OS PRIMEIROS CARACTERES DIGITAIS: um estudo a partir dos computadores pessoais
}

\author{
Roberto Temin \\ FAUUSP \\ rtemin@usp.br \\ Elizabeth Romani \\ FAUUSP \\ elizabethromani@yahoo.com.br \\ Vicente Gil Filho \\ FAUUSP \\ vgil@usp.br
}

Resumo: No final do século $X X$, os designer gráficos adotam uma nova ferramenta de trabalho, o computador pessoal. Entre os vários motivos que explicam essa mudança, há o estudo dos primeiros caracteres digitais, suas características e os aspectos ligados ao design. Este artigo tem como recorte os caracteres dos computadores pessoais produzidos no Brasil do final da década de 1970 a meados dos anos 1980. A compreensão do uso desses caracteres se deu a partir da seleção dos computadores Apple II, de 1977; MSX, de 1983; Sinclair ZX Spectrum, de 1982; IBM PC, de 1981 e o Apple Macintosh, de 1984. A análise dos primeiros caracteres foi embasada pelas características: tamanho da malha de construção dos caracteres, altura do corpo da letra, espessura dos caracteres e presença de serifa.

Palavras-chave: história da tipografia, tipografia digital, caracteres digitais

Abstract: In the late twentieth century, graphic designers adopt a new working tool, the personal computer. Among the several reasons that help understand this transformation, is the study of the personal computer's first digital characters. This article focuses on the characters of computers produced in Brazil from the late 1970s to the mid 1980s. Apple II, IBM PC, Sinclair Spectrum SX, MSX, and Apple Macintosh. The analysis of these characters was based on the grid size construction, the letter's body height, the thickness of the character and the presence of serifs.

Keywords: history of tipography, digital tipography, digital characters 


\section{INTRODUÇÃO}

O termo "computador pessoal" surgiu em 1957, com o IBM 610 Auto-Point Computer (figura 1), devido ao fato de ser operado por apenas uma pessoa com o uso de um teclado. Nesse momento, o computador pessoal estava dissociado do significado de portabilidade, pois possuía o tamanho de uma mesa de escritório grande e custo elevado. Esse modelo de computador era vendido por U\$55.000,00, de forma que somente empresas tinham recursos para comprá-lo.
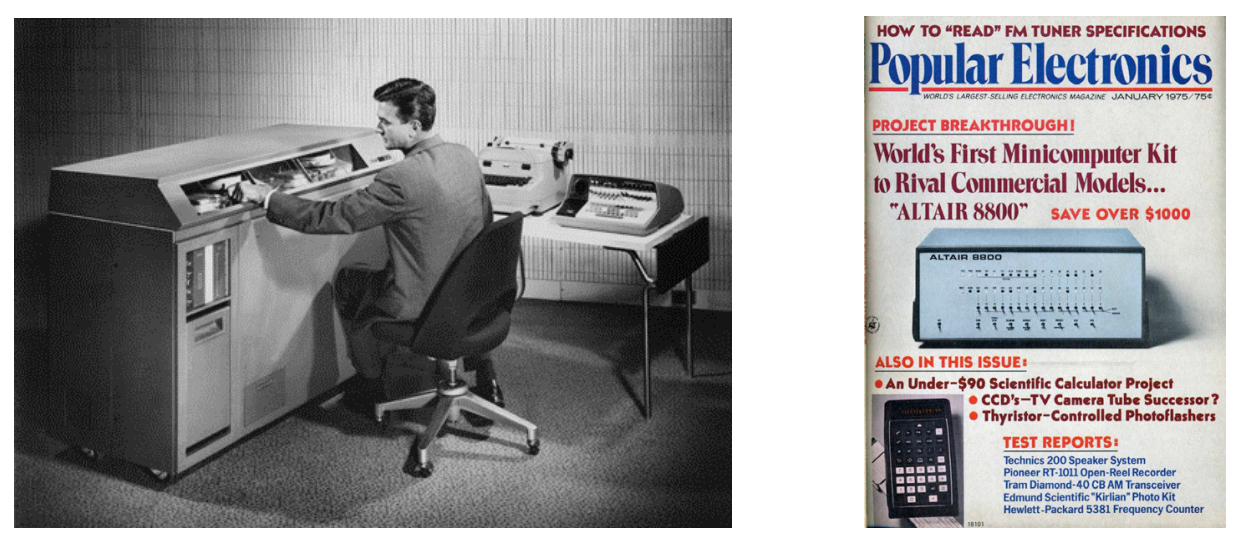

Figura 1 - IBM 610.

Fonte: http://www.computerhistory.org/collections/catalog/102635765

Figura 2 - A revista Popular Electronics com o ALTAIR 8800 na capa.

Fonte: http://www.swtpc.com/mholley/PopularElectronics/Jan1975/PE_Jan_1975_Cover.jpg

Na década de 1970, a miniaturização dos circuitos integrados e a invenção do microprocessador, em 1971 (BETKER, 1997: p.29), possibilitaram a construção dos computadores pessoais como o entendemos hoje. Em janeiro de 1975, a revista americana Popular Electronics mostrava, em reportagem de capa, o Altair 8800, um dos primeiros computadores de uso pessoal (figura 2). Após três meses da publicação, "4 mil pedidos chegaram para a empresa, demonstrando um surpreendente mercado para computadores domésticos" (SWEDIN, 2005: p. 87). O Altair 8800 tinha um custo bem mais acessível ao consumidor: por $U \$ 400,00$ era enviado pelo correio em um "kit" para montar, sem teclado ou monitor.

A partir dessa data, uma série de fabricantes surgiram e desapareceram, todos lutando por um espaço na casa ou no escritório de um usuário que ainda não sabia muito bem para que serviam esses equipamentos.

Era necessário uma aplicação que fosse útil, tal como um processador de texto ou uma planilha eletrônica. O Apple II incorpora essas necessidades de mercado, lançando os programas EasyWriter e Visicalc, ambos de 1979. O Visicalc foi o primeiro programa de planilha desenvolvido para um computador pessoal e considerado um killer $a p p^{1}$, atraindo empresas e fazendo do Apple II um grande sucesso de vendas. Isso chamou a atenção da IBM (BETKER, 1997: p.37), que criou o seu PC (Personal Computer) em 1981.

\footnotetext{
${ }^{1}$ Programa que aumenta significativamente a venda de um computador específico. O Visicalc fez crescer as vendas do Apple II. (SWEDIN, 2005: p. 93)
} 


\title{
2. PARÂMETROS PARA ANÁLISE DOS CARACTERES
}

De acordo com o sistema de classificação adotado pela Associação Tipográfica Internacional (Vox-Atypl), as fontes dos primeiros computadores pessoais a seguir analisadas podem ser classificadas como lineares ${ }^{2}$ ou mecânicas ${ }^{3}$. Uma das características para análise, que deve ser levada em conta, é a malha de pixels que gera o caractere na tela desses computadores. Essa malha é muito pequena, o que torna difícil diferenciar seu desenho com tão pouca informação visual.

Zuzana Licko, cofundadora da Emigre $^{5}$ com Rudy VanderLans e uma das primeiras designers a se ocupar com tipografia digital, lembra da impossibilidade de adaptar a fonte Goudy Old Style com um número tão reduzido de pixels:

\begin{abstract}
I started my venture with bitmap type designs, created for the coarse resolutions of the computer screen and dot matrix printer. The challenge was that because the early computers were so limited in what they could do you really had to design something special (...) it was physically impossible to adapt 8-point Goudy Old Style to 72 dots to the inch. In the end you couldn't tell Goudy Old Style from Times Roman or any other serif text face. (...) It is impossible to transfer typefaces between technologies without alterations because each medium has its peculiar qualities and thus requires unique designs. ${ }^{6}$
\end{abstract}

Apesar da dimensão reduzida da malha de pixels, é possível quantificar os caracteres pela largura total, altura total, altura-x, espessura das hastes e a existência de serifas (figura 3 ).
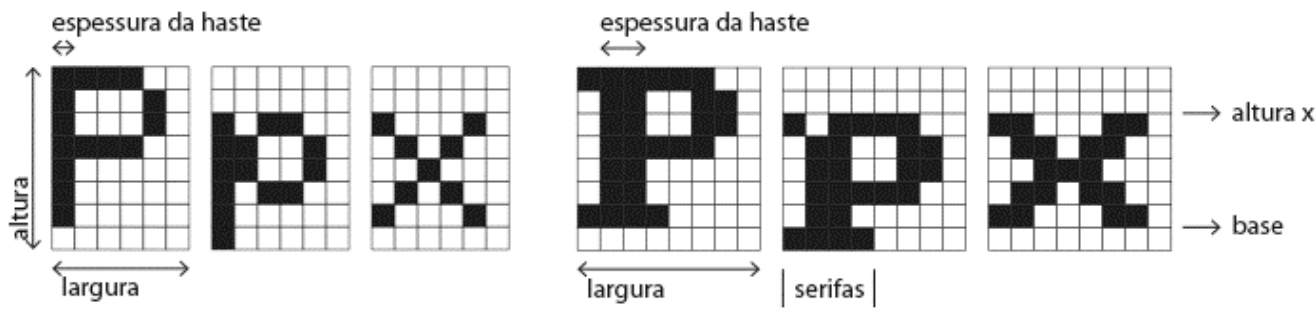

Figura 1 - características dos caracteres

Fonte: Elaborado pelos autores, com base na pesquisa realizada.

\footnotetext{
${ }^{2}$ As fontes lineares não possuem serifas ou variações de espessura das hastes.

${ }^{3}$ As fontes mecânicas têm formas geométricas, serifas e pouca ou nenhuma variação de espessura nas hastes.

${ }^{4}$ Pixel é o menor elemento de uma imagem digital. O nome pixel vem da contração da expressão em inglês picture element, que significa "elemento da imagem".

${ }^{5}$ Emigre é uma empresa que cria fontes digitais, fundada em 1984 no norte da Califórnia, EUA. Foi uma das primeiras font houses independentes a se estabelecer com a construção e o uso de fontes pelo computador pessoal. Também reconhecida pela publicação da revista de design Emigre Magazine entre 1984 e 2005.
}

${ }^{6}$ Comecei o meu negócio com desenhos de tipo bitmap, criados para as baixas resoluções da tela do computador e da impressora matricial. Os primeiros computadores eram tão limitados que o desafio era projetar algo realmente especial (...) foi fisicamente impossível adaptar uma fonte Goudy Old Style de altura 8 pontos para uma resolução de 72 pontos por polegada. No final, você não conseguia diferenciar a Goudy Old Style da Times Roman ou de qualquer outro texto serifado. (...) É impossível transferir as fontes entre tecnologias diferentes sem alteração no desenho, cada tecnologia tem suas peculiaridades e requerem diferentes designs. (VANDERLLANS, 1994: 18-23) - tradução livre. 
O conjunto de caracteres dos primeiros computadores pessoais era definido pelo Código Norte-Americano Padronizado de Intercâmbio de Informações (American Standard Code for Information Interchange ou ASCII), que permitia somente 128 caracteres, não possuindo suporte para acentuação em língua portuguesa. A partir da década de 1980, o padrão ASCII foi estendido para aceitar até 256 caracteres.

Para a análise dos caracteres foram levadas em conta as seguintes características: 1 . Tamanho da malha de construção dos caracteres; 2 . Altura do corpo da letra; 3. Espessura dos caracteres; 4. Presença ou não de serifa.

No caso do Apple Macintosh, para comparação foi analisada a fonte Chicago, com espaçamento proporcional, diferente dos sistemas anteriores, em que os caracteres tinham tamanho e espaçamento fixo.

A análise é feita por meio do modo texto, em que a tela é dividida em uma malha de pixels para a formatação do caractere. A quantidade total de caracteres, colunas e linhas em um monitor depende do tamanho da malha de pixels e da resolução de tela de um determinado computador (tabela 1 ).

Tabela 1 - Quantidade, tamanho da malha de pixels e resolução de tela.

\begin{tabular}{|l|l|l|l|}
\hline Modelo & $\begin{array}{l}\text { Colunas x linhas } \\
\text { de caracteres }\end{array}$ & $\begin{array}{l}\text { Malha do } \\
\text { caractere }\end{array}$ & $\begin{array}{l}\text { Resolução de } \\
\text { tela }\end{array}$ \\
\hline Apple II & $40 \times 24$ & $7 \times 8$ & $280 \times 192$ \\
\hline MSX & $40 \times 24$ & $6 \times 8$ & $256 \times 192$ \\
\hline ZX Spectrum & $32 \times 24$ & $8 \times 8$ & $256 \times 192$ \\
\hline IBM PC $^{7}$ & $\begin{array}{l}40 \times 25 \\
80 \times 25\end{array}$ & $\begin{array}{l}8 \times 8 \\
8 \times 8\end{array}$ & $\begin{array}{l}320 \times 200 \\
640 \times 200\end{array}$ \\
\hline Apple Macintosh & variável & variável & $512 \times 342$ \\
\hline
\end{tabular}

Fonte: Elaborado pelos autores, com base na pesquisa realizada.

\subsection{Especificidade dos clones brasileiros}

Os computadores fabricados no Brasil em meados da década de 1980 eram clones dos estrangeiros, como por exemplo, o Apple II e o IBM PC. A versão copiada dos computadores possuía a desvantagem da ausência de acentuação. Um dos clones do Apple II, o TK3000, da Microdigital, tinha teclado e caracteres acentuados, mas isso ocasionava problemas de compatibilidade em alguns programas, e para impressão com acentos era necessário uma placa de expansão específica.

O teclado do Scopus Nexus 1600, primeiro compatível com o IBM PC, não possuía cedilha ou acentuação, que era construída por combinações de teclas. Os acentos tinham que ser feitos por programação, como explica Eugênio Vilar ${ }^{8}$, criador

\footnotetext{
${ }^{7}$ A placa de vídeo CGA, de 1981, foi o primeiro padrão gráfico utilizado no IBM PC e suportava dois modos de texto, 40 e 80 colunas, utilizando a mesma malha de $8 \times 8$ de caracteres.

8 Eugênio Villar é mestre pelo Institute of Computer Science da Universidade de Londres. Trabalhou na Burroughs Brasileira na década de 1970 e para a Cobra - Computadores Brasileiros S.A. - a partir de 1979. Atualmente é analista de sistemas no Rio de Janeiro.
} 
do editor de texto Pangloss, para PC, em meados de 1980: "A acentuação ocorre durante a digitação do texto, quando a máscara do acento se sobrepõe à máscara da letra através de um 'ou lógico' binário (...) o texto é literalmente desenhado por programa, um pixel de cada vez."

\section{ANÁLISE DOS CARACTERES DOS COMPUTADORES PESSOAIS}

\subsection{Apple II}

Fabricado a partir de 1977 pela Apple Inc., o Apple II foi um dos primeiros computadores pessoais com grande sucesso de vendas nos Estados Unidos. Esse foi um dos computadores de maior longevidade dessa indústria, sendo descontinuado apenas em 1993. Por sua grande popularidade e uma vasta biblioteca de programas, o Apple II foi clonado no Brasil. O Microengenho, da empresa Spectrum; o Exato Pro, da CCE; o TK3000 Ile, da Microdigital, e o D-8100, da Dismac, foram alguns dos clones brasileiros.

Seu conjunto de caracteres (figura 4) não apresenta serifa. A malha do caractere é formada por 7 pixels na horizontal e 8 pixels na vertical (figura 5). A caixa alta ultrapassa a altura da linha ascendente, encostando no topo da malha, o que faz com que as letras maiúsculas e os pontos da letra i e j encostem nas descendentes das letras $\mathbf{g}, \mathbf{j}, \mathbf{p}$, q e y, dificultando a leitura entre linhas (figura 6). As hastes têm 1 pixel de espessura, com exceção dos colchetes e das chaves, que têm 2 pixels.

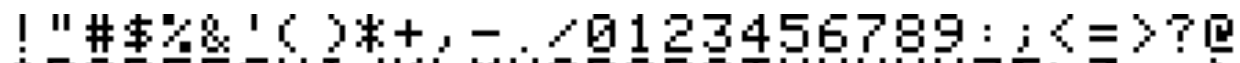

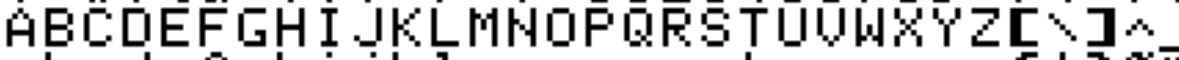

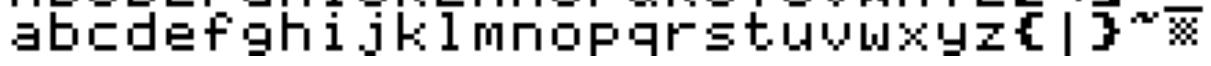

Figura 4 - Conjunto de caracteres do Apple II.

Fonte: Elaborado pelos autores, com base na pesquisa realizada.

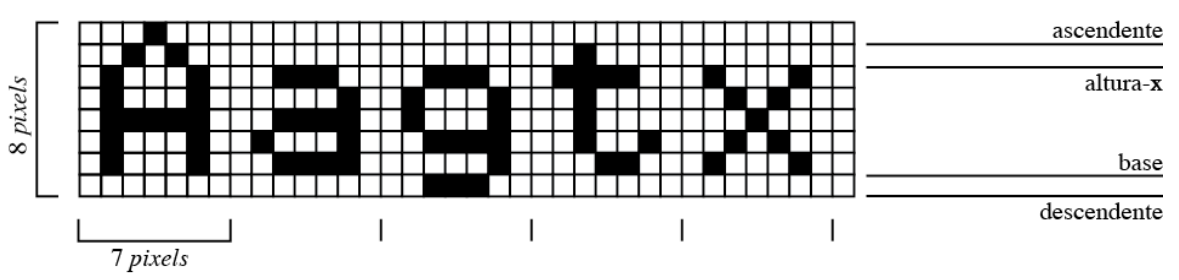

Figura 5 - Malha de caracteres do Apple II.

Fonte: Elaborado pelos autores, com base na pesquisa realizada.

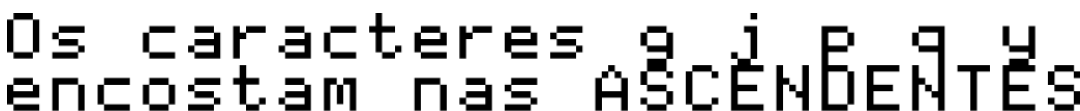

Figura 6 - Exemplo dos caracteres g, j, p, q e y que encostam nas ascendentes.

Fonte: Elaborado pelos autores, com base na pesquisa realizada. 


\subsection{MSX}

Criado em 1983, no Japão, a versão brasileira do MSX foi produzida e comercializada por duas empresas: a partir de 1985 pela Sharp, com o modelo HotBit, descontinuado em 1988, e pela Gradiente, com o Expert, em várias versões até 1991. O MSX foi popular no Brasil como computador doméstico e plataforma para jogos. Ambos tinham acentuação em português, porém suas tabelas de caracteres não eram padronizadas (figura 7), fazendo com que não fossem $100 \%$ compatíveis entre si, com teclas em posições diferentes e com diferenças na acentuação.

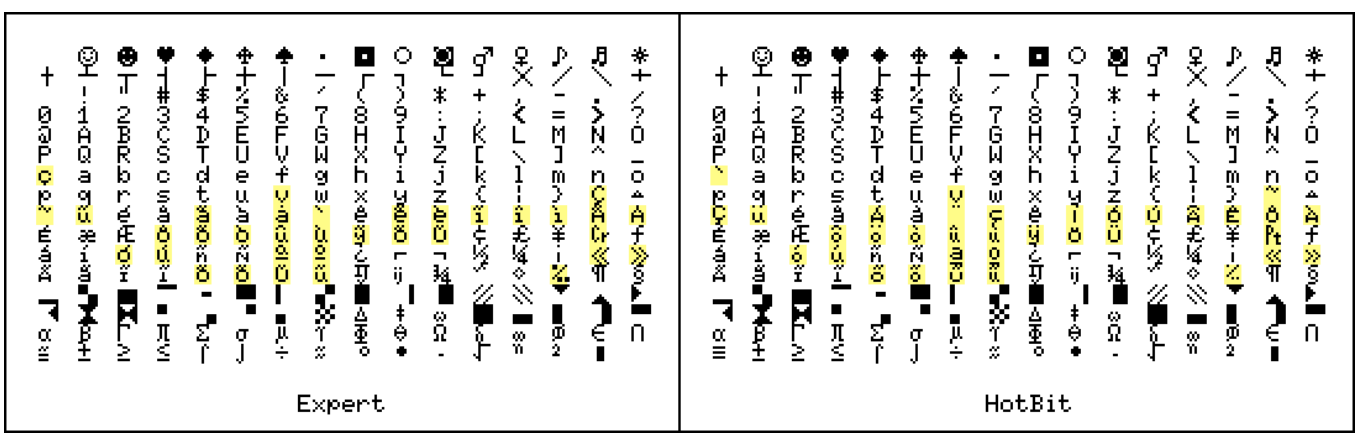

Figura 7 - Diferenças dos glifos do MSX Expert, da Gradiente, e do MSX HotBit, da Sharp.

Fonte: Elaborado pelos autores, com base na pesquisa realizada.

O conjunto de caracteres não apresenta serifa (figura 8). A malha do caractere é formada por 6 pixels na horizontal e 8 pixels na vertical (figura 9). A caixa alta e a linha ascendente encostam no topo da malha, o que faz com que as letras maiúsculas, as letras ascendentes e as letras acentuadas ou com ponto, como as letras $\mathbf{i}$ e $\mathbf{j}$, encostem nas descendentes das letras $\mathbf{g}, \mathbf{j}, \mathbf{p}, \mathbf{q}$ e $\mathbf{y}$, prejudicando a leitura entre linhas. Possuem hastes com 1 pixel de espessura.

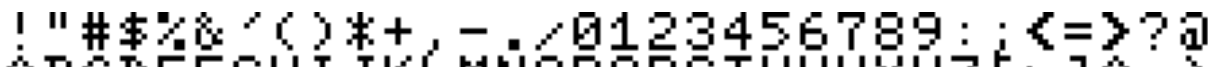

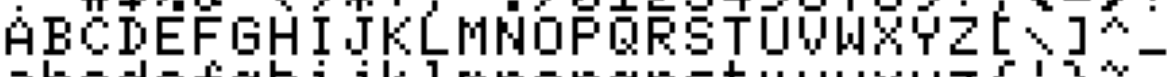 abodefghi jkl mnoparstuvwxuz}

Figura 8 - Conjunto de caracteres básico de um MSX.

Fonte: Elaborado pelos autores, com base na pesquisa realizada.

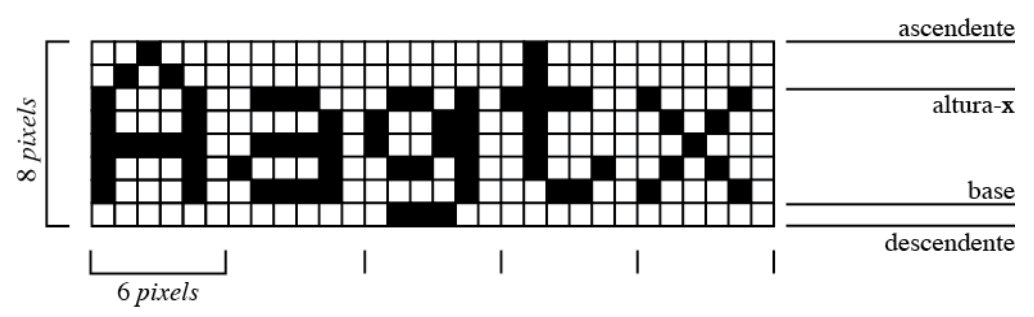

Figura 9 - Malha de caracteres do MSX.

Fonte: Elaborado pelos autores, com base na pesquisa realizada.

\subsection{ZX Spectrum}

Computador inglês desenvolvido e comercializado pela Sinclair Research Ltda. a partir de 1982 foi descontinuado em 1992. No Brasil foi clonado pela Microdigital com os modelos TK-90X, de 1985, e TK95, de 1986. O computador nacional não era $100 \%$ compatível com o original, no entanto, a maioria dos programas e jogos funcionavam. 
O conjunto de caracteres não apresenta serifa (figura 10). A malha do caractere é formada por 8 pixels na horizontal e 8 pixels na vertical (figura 11). O modelo brasileiro acrescentou caracteres acentuados acessíveis por tecla de função, pois o teclado, cópia idêntica do computador inglês, não continha acentos ou cedilha.

A caixa alta tem a mesma altura que a linha ascendente, não compro-metendo a leitura entre linhas. Possuem hastes com 1 pixel de espessura.

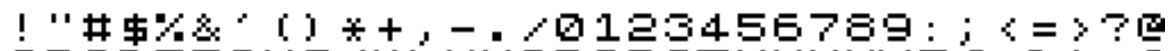

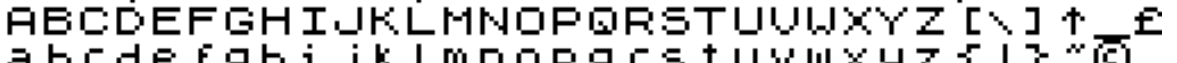

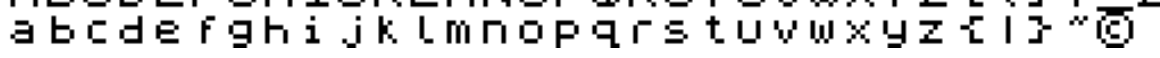

Figura 10 - Conjunto de caracteres do ZX Spectrum.

Fonte: Elaborado pelos autores, com base na pesquisa realizada.

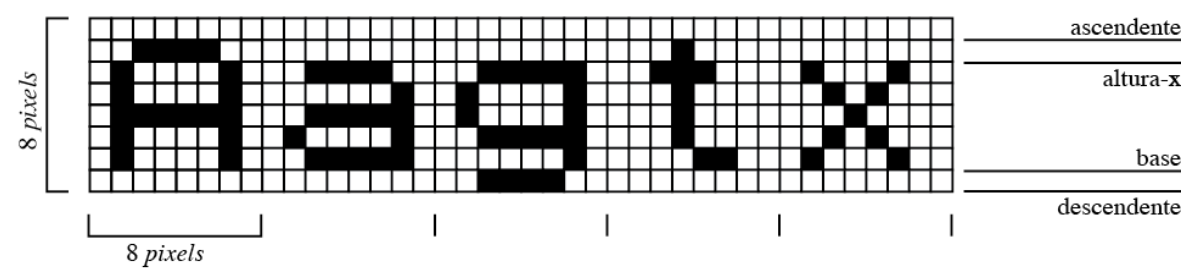

Figura 11 - Malha de caracteres do ZX Spectrum.

Fonte: Elaborado pelos autores, com base na pesquisa realizada.

\subsection{IBM PC}

Computador pessoal criado pela IBM em 1981. Sua arquitetura aberta permitiu que fosse copiado no mundo inteiro, inclusive no Brasil. No final da década de 1980, torna-se a arquitetura de computadores pessoais dominante, tanto no mercado corporativo quanto no doméstico. Entre as cópias brasileiras destacam-se o Nexus 1600 da Scopus o primeiro clone nacional do IBM PC, o i7000 PC-XT da Itautec e o semiportátil Solution 16 da Prológica.

O conjunto de caracteres apresenta serifas (figura 12 e 13). O padrão ASCll estendido não continha todos os acentos em português, os caracteres a e o não tinham "til" e nem acento agudo (figura 14). A malha do caractere é formada por 8 pixels na horizontal e 8 pixels na vertical (figura 15).

A caixa alta e a linha ascendente encostam no topo da malha, isso faz com que as letras maiúsculas, as letras ascendentes e as letras acentuadas ou com ponto, como as letras i e j, encostem nas descendentes das letras $\mathbf{g}, \mathbf{j}, \mathbf{p}, \mathbf{q}$ e $\mathbf{y}$, prejudicando a leitura entre linhas. Possuem hastes com 2 pixels de espessura.

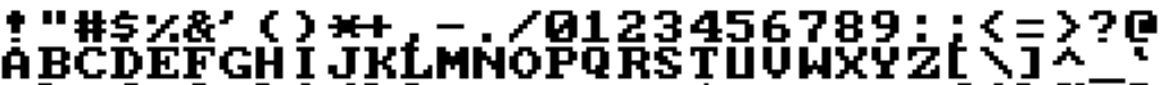

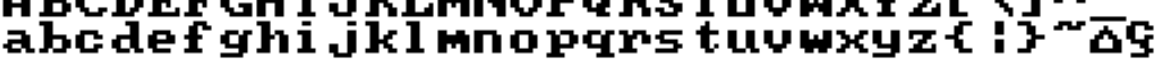

Figura 12 - Conjunto de caracteres do IBM PC no padrão CGA de 40 colunas.

Fonte: Elaborado pelos autores, com base na pesquisa realizada.

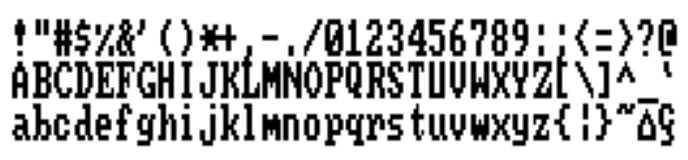

Figura 13 - Conjunto de caracteres do IBM PC no padrão CGA de 80 colunas. Fonte: Elaborado pelos autores, com base na pesquisa realizada. 


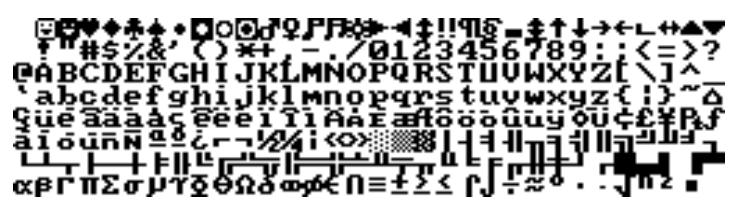

Figura 14 - Conjunto de glifos suportados pelo IBM PC.

Fonte: Elaborado pelos autores, com base na pesquisa realizada.

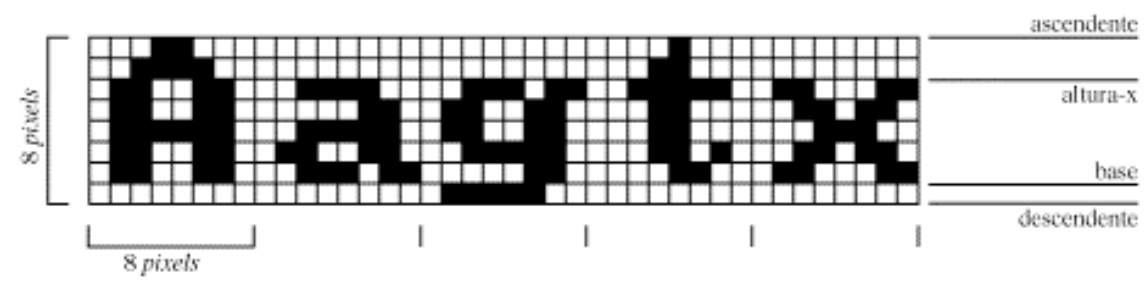

Figura 15 - Malha de caracteres do IBM PC no padrão CGA.

Fonte: Elaborado pelos autores, com base na pesquisa realizada.

\subsection{Apple MACINTOSH}

Lançado em 1984, foi o primeiro computador pessoal com um sistema operacional usando uma interface gráfica e uso do mouse.

Chegou a ser clonado no Brasil pela empresa Unitron em 1986. Em 1987, a Apple e o Departamento do Estado norte-americano, alegando pirataria, pressionaram o governo brasileiro, que proibiu, em 1988, a fabricação nacional desse computador.

Com uma tela integrada de 9 polegadas com densidade de 72 pixels por polegada, cada pixel correspondia a 1 ponto. O Macintosh vinha com 9 fontes: Chicago, New York, Geneva, Monaco, Cairo, London, Los Angeles e San Francisco, desenhadas por Susan Kare, e Venice, desenhada por Bill Atkinson (figura 16).

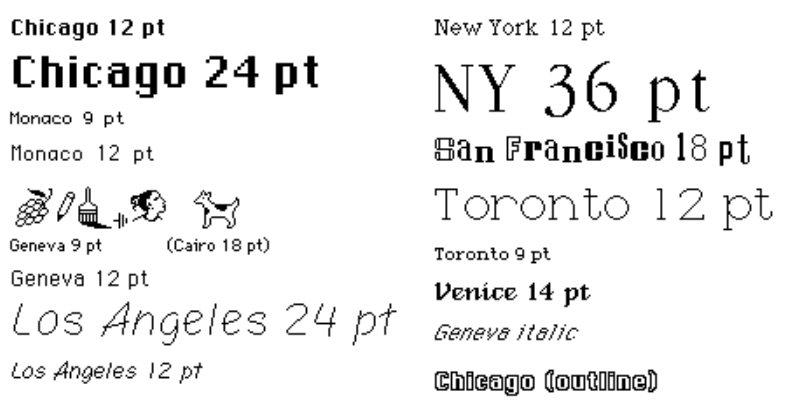

Figura 16 - Fontes incluídas no sistema operacional.

Fonte: Elaborado pelos autores, com base na pesquisa realizada.

Foi também o primeiro computador a ter fontes proporcionais, como cita Susan Kare, designer das fontes e dos ícones do primeiro sistema operacional do Macintosh:

My career in user interface graphic design began when I worked for Apple Computer between 1983 and 1986. My job: icon and font designer for a new computer, the Macintosh. The task: to transform small grids of black and white pixels into a family of symbols that would assist people in operating the computer.(..) My work also focused on developing a set of proportional typefaces for the computer screen; a departure from the 
monospaced characters typically found on typewriters and earlier computers. ${ }^{9}$

Uma letra com doze pontos de altura usava até 12 pixels verticais e um número de pixels variável na horizontal. A fonte aqui analisada é a Chicago no corpo 12, que foi usada nos menus da interface gráfica. Os caracteres em um programa de editoração de texto, por exemplo, poderiam ser vistos na tela com o mesmo tamanho que na impressão. A expressão What You See Is What You Get foi usada para indicar essa similaridade.

O conjunto de caracteres não apresenta serifa (figura 17). A malha do caractere é formada por um número variável de pixels na horizontal e 12 pixels na vertical (figura 18). Nesse caso a letra $\mathbf{i}$, mais estreita, tem 2 pixels de espessura, e a $\mathbf{m}$, a mais larga, tem 10 pixels. A distância entre linhas é variável, não se aplicando as restrições dos sistemas anteriores. A linha ascendente não encosta na linha descendente. Possuem hastes com 2 pixels de espessura.

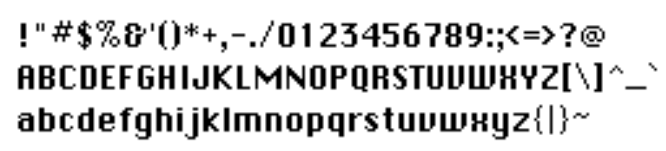

Figura 17 - Conjunto de caracteres da fonte Chicago.

Fonte: Elaborado pelos autores, com base na pesquisa realizada.
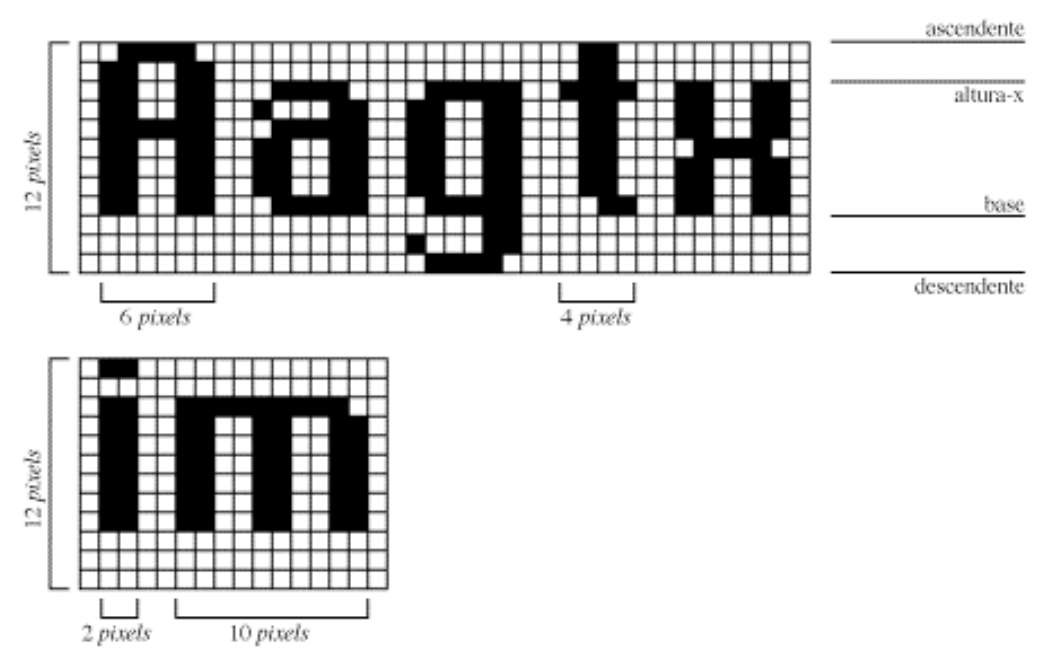

Figura 18 - Malha de caracteres da fonte Chicago.

Fonte: Elaborado pelos autores, com base na pesquisa realizada.

\footnotetext{
${ }^{9}$ Minha carreira em design de interface começou quando eu trabalhava para a Apple Computer, entre 1983 e 1986. Meu trabalho: projetar ícones e fontes para um novo computador, o Macintosh. A tarefa: transformar pequenas malhas de pixels preto e branco em uma família de símbolos que ajudassem as pessoas a utilizar o computador.(...) Meu trabalho também se concentrou no desenvolvimento de um conjunto de tipos proporcionais para a tela do computador, diferente dos caracteres monoespaçados tipicamente encontrados em máquinas de escrever e computadores daquela época. - tradução livre. (http://www.kare.com/design_bio.html)
} 


\title{
4. NOVAS POSSIBILIDADES
}

Ao analisar os primeiros caracteres, antes de 1984, nota-se que seu desenho para a tela do computador pessoal, não era uma prioridade, e sim algo que tinha que ser feito para solucionar uma necessidade, mas sem a preocupação com o desenho das letras ou sua legibilidade, uma vez que também era difícil projetar uma família tipográfica em uma malha de pixels limitada, devido a resolução dos computadores. Rupert Goodwins, um programador da empresa de tecnologia inglesa Sinclair Research no início da década de 1980 e editor da revista inglesa ZDNet até 2012, escreveu:

\begin{abstract}
I was a font designer for Sinclair Research... actually, I was about the lowest of the low in the software engineering pecking order and happened to be doing the screen handling software for a new product, so I got to do the font as well.(...) I can't speak for any of the other companies, but 'font design' is perhaps the wrong term for the process. Much faffing with graph paper, lots of over-the-shoulder critiquing from other software engineers, but not a high priority nor something that was seen as needing any particular graphic or typographic skills. ${ }^{10}$
\end{abstract}

De acordo com Ferreira (2004, p.86-87), "fontes criadas especialmente para mídia em tela, construídas a partir de módulos quadrados que correspondem aos pixels do monitor, podem ser usadas apenas no tamanho específico para o qual foram criadas". Esses caracteres, inseridos na memória permanente do computador, eram os únicos disponíveis até o surgimento, em 1984, do computador Apple Macintosh e, em 1985, da linguagem PostScript ${ }^{11}$ da Adobe.

Essas primeiras fontes serviam basicamente para a interface homem-máquina, sem preocupações estilísticas, uma vez que a malha geradora desses caracteres era muito pequena, não permitindo variações de forma e espessura. Afirma Farias (1997, p.68) que, "nos primeiros computadores pessoais (...), o desenho de fontes era limitado pela baixa resolução das telas e impressoras. As letras deveriam ser necessariamente desenhadas a partir de bitmaps, resultando em contornos visivelmente serrilhados".

Até 1984 os computadores pessoais recebiam tarefas por meio de uma linha de comando (figura 19). O Apple Macintosh foi o primeiro computador pessoal a apresentar uma interface gráfica (figura 20) com o uso do mouse.

Essa revolução na interface ampliou o uso do computador pessoal como ferramenta de trabalho inovadora para área gráfica.

\footnotetext{
${ }^{10}$ Eu era o designer de fontes para a Sinclair... na verdade, eu era o que tinha a menor qualificação na engenharia de software da empresa e fui designado para fazer o software de manipulação de tela para um novo produto e, por conta disso, também tive que fazer o tipo de letra. Não posso falar de outras empresas, mas design de fontes talvez não seja o termo correto para o processo, com muitos desenhos em papel quadriculado, muitas opiniões de outros engenheiros. Não era uma prioridade, nem algo que tinha que ser feito por alguém com conhecimento específico em design gráfico ou tipográfico. tradução livre. (http://damieng.com/ blog/2011/02/20/typography-in-8-bits-system-fonts)

${ }^{11}$ PostScript é uma linguagem de descrição de página criada inicialmente para impressão de arquivos de alta qualidade, independente de resolução. Com a incorporação dessa linguagem em programas gráficos, os caracteres gerados podiam ser ampliados ou reduzidos mantendo a qualidade de impressão.
} 


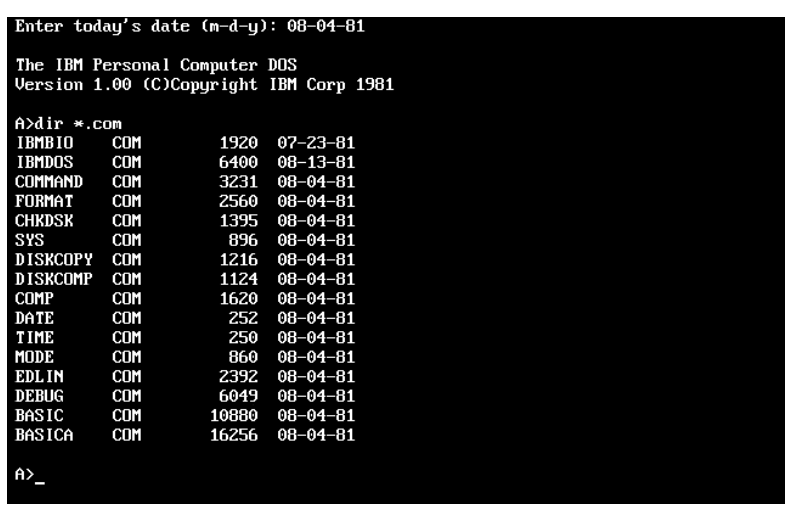

Figura 19 - Linha de comando do MS-DOS para IBM PC e compatíveis.

Fonte: http://www.quepublishing.com/articles/article.aspx?p=1358665

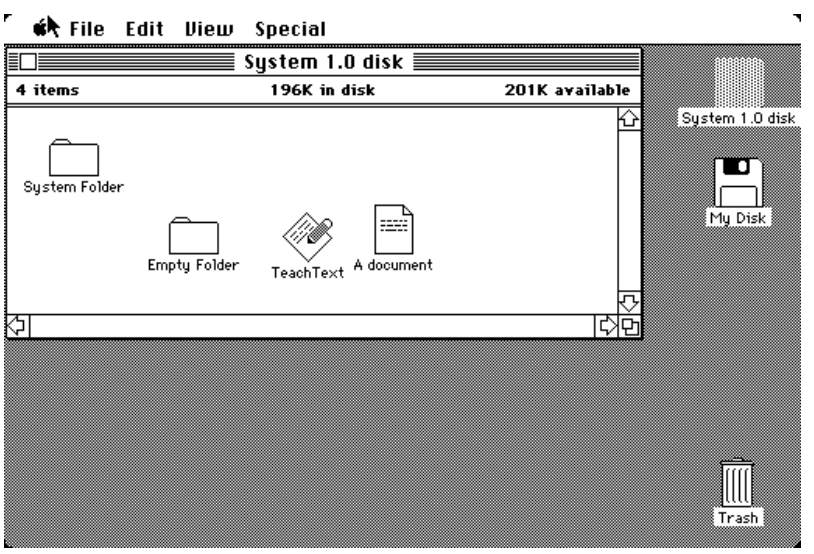

Figura 20 - Interface gráfica do Apple Macintosh de 1984.

Fonte: http://www3.nd.edu/ jvanderk/sysone/

\section{CONSIDERAÇÕES FINAIS}

A primeira geração dos computadores pessoais, no final da década de 1970, possuía uma malha bitmap que não passava de $8 \times 8$ pixels, limitando o desenho dos diferentes caracteres a pequenas variações. A distância entre as letras não variava, fazendo com que o espaço entre os caracteres " $i$ " e " $\mathrm{l}$ " aparentasse uma distância maior do que as demais. Os caracteres " $g$ ", "j", "p", "q" e " $\mathbf{~ " ~ m u i t a s ~ v e z e s ~}$ encostavam na linha ascendente abaixo, diminuindo sua legibilidade.

$\mathrm{Na}$ década seguinte, com o surgimento dos sistemas operacionais gráficos, todos os pixels da tela são manipuláveis, não sendo mais necessário uma malha específica para os caracteres.

O estudo dos caracteres gerados pelos primeiros computadores pessoais faz parte do entendimento a respeito de como, a partir da criação dessas máquinas, os vários aspectos do design gráfico, e da tipografia em particular, foram evoluindo e adaptando-se a esse universo. Em meados da década de 1980, o surgimento do Macintosh, da Apple, e do Microsoft Windows para PCs, proporcionou uma grande facilidade de uso e tornou estes equipamentos amigáveis. Desta forma cada vez mais designers gráficos se interessaram em utilizar tais equipamentos, iniciando um ciclo de constantes transformações e solidificando o uso do computador pessoal como ferramenta de trabalho do campo gráfico. 


\section{REFERÊNCIAS}

CHANDLER, Scott Bondurant. Comparing the legibility and comprehension of type size, font selection, and rendering technology of onscreen type. Virginia Polytechnic Institute and State University, 2001.

BETKER, Michael R., et. al. The History of the Microprocessor. Bell Labs Technical Journal, 1997.

BOMENY, Maria Helena Werneck. O Panorama do design gráfico contemporâneo: a construção, a descontrução e a nova ordem. Tese (doutorado) - Universidade de São Paulo, Curso de Pós-Gradução em Design e Arquitetura, 2009.

BERNING, Thomas J. (Oct-Dec 2006). "The Origins of Word Processing Software for Personal Computers: 1976-1985". In: IEEE Annals of the History of Computing, Anais: volume 28 , issue 4.

FARIAS, Priscila Lena. Tipografia na era digital: o impacto das novas tecnologias. Dissertação (mestrado) - Pontifícia Universidade Católica, Curso de Pós-Graduação, 1997.

FERREIRA, Gustavo. Universo bitmap em evolução. In: Tupigrafia. ano 3, n.5, p.86-87 São Paulo, julho 2004.

FRIEDRICH A. KITLER. Gramophone, film, typewriter. Stanford , California: Stanford University Press, 1999.

GIL, Vicente. A Revolução dos Tipos. Tese (doutorado) - Universidade de São Paulo, Curso de Pós-Gradução em Design e Arquitetura, 1999.

IBM (1983). Personal Computer Hardware Reference Library: Guide to Operations, Personal Computer XT. IBM Part Number 6936831.

LEGGE, Gordon E. e BIGELOW, Charles A. Does print size matter for reading? A review of findings from vision science and typography. 2011.

LUZ, Filipe Costa. Design digital: do carvão ao pixel. Departamento de Ciências da Comunicação, Artes e Tecnologia da Informação, Univ. Lusófona de Humanidades e Tecnologias. 2003. Disponível em: http://hdl.handle.net /10437/2680. Acesso em 10 jul. 2013.

SEARLE, Steven J. A Brief History of character codes in North America, Europe, and East Asia. Sakamura Laboratory, University Museum, University of Tokyo Disponível em: http://tronweb.super-nova.co.jp/characcodehist.html Acesso 15 jul.2013.

SWEDIN, Eric G., FERRO, David L. Computers: The Life Story of a Tecnology. The Johns Hopkins University Press, 2007.

VANDERLLANS, Rudy, LICKO, Zuzana, GREY, Mary. Emigre, Graphic design into the digital realm. Van Nostrand Reinhold Company, 1994.

WEISENMILLER, Eric Michael. A study of the readability of on-screen text. 1999. Disponível em: http://scholar.lib.vt.edu/theses/available/etd-102999110544/unrestricted/WeisenmillerDissertation.pdf - Acesso 15 jul. 2013.

VILAR, Eugênio. Entrevista concedida por e-mail, em fevereiro de 2013. 